\title{
The Need for Orthodontic Treatment among Vietnamese School Children and Young Adults
}

\author{
Son Minh Nguyen, ${ }^{1}$ Minh Khac Nguyen, ${ }^{1}$ Mare Saag, ${ }^{2}$ and Triin Jagomagi ${ }^{2}$ \\ ${ }^{1}$ Da Nang University of Medical Technology and Pharmacy, 99 Hung Vuong, Da Nang 59000, Vietnam \\ ${ }^{2}$ Department of Stomatology, University of Tartu, Raekojapl 6, 51003 Tartu, Estonia \\ Correspondence should be addressed to Triin Jagomagi; triin.jagomagi@ortodontia.ee
}

Received 23 March 2014; Revised 3 June 2014; Accepted 26 June 2014; Published 21 July 2014

Academic Editor: James K. Hartsfield

Copyright (c) 2014 Son Minh Nguyen et al. This is an open access article distributed under the Creative Commons Attribution License, which permits unrestricted use, distribution, and reproduction in any medium, provided the original work is properly cited.

Objective. The aim of this study was to evaluate the need for orthodontic treatment among 12-year-old school children and 18-yearolds from Da Nang, Vietnam. Basic Research Design. A random representative sample of 20012 -year-old children from primary schools in Da Nang city was gathered. In addition, 200 18-year-old students were randomly selected from among the 4000 students studying at Da Nang University of Medical Technology and Pharmacy, Vietnam. All the subjects were evaluated according to Angle's molar relationship, the presence of malocclusion, and the components of the Index of Orthodontic Treatment Need (Dental Health Component, DHC, and Aesthetic Component, AC). Results. The DHC of index of orthodontic treatment need (IOTN) for 12year-olds was in $60 \%$ of cases no or little, in $21 \%$ of cases moderate, and in $19 \%$ of cases definitive, while the prevalence of moderate and definitive need for treatment among the 18 -year-olds was $24 \%$ and $30.5 \%$, respectively. The prevalence of class III malocclusion, contact point displacement, and crossbite was higher in 18-year-olds than among the 12-year-olds, while the prevalence of increased overjet and increased overbite had decreased in 18-year-olds compared to the group of 12-year-olds. Conclusions. There is a strong need for orthodontic treatment in Vietnam's population. The need for orthodontic treatment was determined by contact point displacement, crossbite, increased overjet, and increased overbite.

\section{Introduction}

The need for orthodontic treatment is influenced by a number of factors including cultural, parental, peer, and selfperception of dental beauty. The assessment of malocclusion and treatment need for public health purposes is crucial for planning orthodontic care service in terms of planning necessary human and financial resources, and also for monitoring the oral health programs offered. Such data are not available for the population of Vietnam.

Some indices have been developed to objectify treatment need. These include the Dental Aesthetic Index (DAI) [1], The California Modification of the Handicapping Labiolingual Deviation Index, the HLD (CalMod) index [2]. The aforementioned indices are useful to determine resource allocation in particular populations or communities, to select those patients who can be treated in a particular dental care system, and to establish priorities when resources are limited.
Brook and Shaw [3] introduced the Index of Orthodontic Treatment Need (IOTN). The IOTN classifies malocclusions according to the presence of particular occlusal features considered important for dental health and aesthetics in order to identify individuals who would benefit most from orthodontic treatment. The IOTN has two separate components: a clinical component referred to as the Dental Health Component (DHC) and Aesthetic Component (AC); DHC with five severity levels and an AC with ten severity levels. There was no attempt to combine these two components and both are recorded separately. The IOTN has been used for this purpose in many epidemiological studies.

Assessment of orthodontic treatment need and demand helps in planning orthodontic services. In Malaysia [4], $47.9 \%$ of school children had a definite need for orthodontic treatment, and the percentage was $37 \%$ among Hong Kong Chinese [5]. Livas and Delli [6] systematically reviewed the literature concerning orthodontic treatment need and 
established that the prevalence of definite need of DHCIOTN is often greater than $11 \%$, and extends up to $70 \%$, although results are affected by gender, age, and region.

Vietnam is the easternmost country on the Indochina Peninsula in Southeast Asia; Da Nang is the biggest city on the South Central Coast of Vietnam and is the commercial and educational center of Central Vietnam. Little published information is available about the oral health status of the population of Vietnam. The present study aimed to evaluate the need for orthodontic treatment among 12-year-old Vietnamese school children and 18-year-old young adults. The findings may be of value for providing orthodontic and human resource training, treatment facilities, and resource planning.

\section{Material and Methods}

The investigation targeted 200 12-year-old children, who were randomly selected from five primary schools and 200 18-year-old students, randomly selected from among the 4000 students studying at Da Nang University of Medical Technology and Pharmacy. The subjects had no history of orthodontic treatment. A minimum sample size consisting of 384 individuals was calculated on the premise of a prevalence of $50 \%$ for unknown orthodontic treatment need in Da Nang, with a standard error of $5 \%$ and a $95 \%$ confidence interval. A round study sample was set at 400 subjects. The study was performed according to the guidelines of the Research Ethics Committee of the Da Nang University of Medical Technology and Pharmacy.

School children were examined in the dental clinics of their primary schools and the students were examined in the dental clinic of Da Nang University of Medical Technology and Pharmacy without external interference. The examination lasted approximately 15 minutes per participant, following the World Health Organization (1997) guidelines. The assessment of dental occlusion was carried out using latex gloves, dental mirrors, and a CPI probe. No radiographs, study casts, or previous written records were used. Two general dentists evaluated Angle's molar relationship, the presence of malocclusion, and components of the IOTN. Intrarater reliability was assessed during the training session; all the examination of DHC and AC score were assessed two times in a group of 20 subjects by the same dentist (the first author) who was supervised by a certified orthodontist; the time interval between examinations was 2 days and Kappa index was utilized and assessed to be 0.86 for DHC and 0.91 for AC score.

Orthodontic variables including Angle's molar relationship and presence of malocclusion (increased overjet, increased overbite, crossbite, and contact point displacement) were evaluated according the World Health Organization (1997) guidelines. The need for orthodontic treatment was assessed by means of the DHC of the IOTN [3]. The DHC of the IOTN has five grades: grades 4 and 5 represent high priority for treatment, grade 3 is a borderline need, and grades 1 and 2 are little or no need for treatment. The AC consists of ten scaled color photographs showing different levels of dental attractiveness. Grade 1 represents the most attractive tooth arrangement and grade 10 the least attractive arrangement of teeth. When the AC was being recorded, the dental attractiveness of the anterior teeth was graded by an examiner after the subjects had closed their teeth in central occlusion and retracted their lips.

2.1. Analysis. The data were analyzed using version 18.0 SPSS software. Descriptive statistics and the chi square correlations between the orthodontic variables were calculated. Student's $t$-test was used to compare the prevalence of orthodontic treatment need among the two groups. A significance level of $5 \%$ was considered relevant.

\section{Results}

The study encompasses a sample of 200 12-year-old children and 200 students of 18 years of age, with an overall distribution of $54.5 \%$ female and $45.5 \%$ male.

The prevalence of moderate or definite orthodontic treatment need of Dental Health Component scale was recorded in $47.5 \%$ of the sample and Aesthetic Component in $22.8 \%$ of cases (Table 1). The prevalence of a definite need according to the AC scale is higher in females than in males $(P<$ 0.05). There was no statistically significant difference for orthodontic treatment need according to DHC between the age groups $(P>0.05)$.

The prevalence of malocclusion and occlusal traits in the total sample was also recorded. The prevalence of class I, II, and III malocclusion was measured at $67 \%, 17.5 \%$, and $15.5 \%$, respectively. An increased overjet $(>3.5 \mathrm{~mm})$ was present in $36.3 \%$ of subjects while $26.3 \%$ had increased overbite $(>3.5 \mathrm{~mm})$. Contact point displacement was found in $54 \%$ of the subjects examined and $22.3 \%$ had crossbite. A significant difference was observed between malocclusion, increased overjet, crossbite, displacement, and increased overbite between the age groups.

The statistically significant differences of each occlusal trait, involving orthodontic treatment need of DHC, in the total sample are presented in Table $2(P<0.05)$. With subjects showing overjet greater than $3.5 \mathrm{~mm}$, the treatment need was $52.5 \%$, while $78.2 \%$ subjects with contact point displacement of more than $1 \mathrm{~mm}$ were assessed to have an objective need for orthodontic treatment; crossbite and increased overbite were present in $82.4 \%$ and $62.9 \%$, respectively, of cases.

Among the 12 -year-olds, $21 \%$ had a moderate (grade 3 ) and $19 \%$ had a definite (grades 4-5) need for orthodontic treatment (Figure 1). The prevalence of moderate and definite treatment need in the sample of 12-year-olds was lower than in the sample of 18-year-olds.

\section{Discussion}

IOTN was used to evaluate the need for orthodontic treatment and to evaluate the severity of malocclusion. The investigation was performed on subjects from groups of 12and 18-year-olds in Da Nang, Vietnam. According to the DHC of IOTN results, $52.8 \%$ of subjects were in little need of treatment (grades 1-2), 22.5\% had a moderate need for treatment (grade 3), and $24.7 \%$ had a definitive need for treatment (grades 4-5). A statistically significant difference 
TABLE 1: Distribution of molar relationship, AC, DHC, and malocclusion in relation to gender and age group.

\begin{tabular}{|c|c|c|c|c|c|c|c|}
\hline \multirow[b]{2}{*}{ Variables } & \multirow[b]{2}{*}{$n(\%)$} & \multicolumn{2}{|c|}{ Gender } & \multicolumn{4}{|c|}{ Age groups } \\
\hline & & $\begin{array}{c}\text { Female } \\
n(\%)\end{array}$ & $\begin{array}{l}\text { Male } \\
n(\%)\end{array}$ & $P$ value & $\begin{array}{c}12 \\
n(\%)\end{array}$ & $\begin{array}{c}18 \\
n(\%)\end{array}$ & $P$ value \\
\hline \multicolumn{8}{|l|}{ Angle relationship } \\
\hline Class I & $268(67)$ & $158(59)$ & $110(41)$ & 0.79 & $144(72)$ & $124(62)$ & $0.03^{*}$ \\
\hline Class II & $70(17.5)$ & $41(58.6)$ & $29(41.4)$ & 0.99 & $35(17.5)$ & $35(17.5)$ & 1.00 \\
\hline Class III & $62(15.5)$ & $35(56.5)$ & $27(43.5)$ & 0.72 & $21(10.5)$ & $41(20.5)$ & $0.006^{*}$ \\
\hline \multicolumn{8}{|l|}{ Aesthetic Component (AC) } \\
\hline No/little need & $309(77.2)$ & $184(59.5)$ & $125(40.5)$ & 0.43 & $156(78)$ & $153(76)$ & 0.72 \\
\hline Moderate need & $65(16.3)$ & $30(46.2)$ & $35(53.8)$ & $0.02^{*}$ & $35(17.5)$ & $30(15)$ & 0.49 \\
\hline Definite need & $26(6.5)$ & $20(76.9)$ & $6(23.1)$ & $0.04^{*}$ & $9(4.5)$ & $17(8.5)$ & 0.10 \\
\hline \multicolumn{8}{|l|}{ Dental Health Component (DHC) } \\
\hline No/little need & $211(52.8)$ & $115(54.5)$ & $96(45.5)$ & 0.86 & $120(60)$ & $91(45.5)$ & $0.04^{*}$ \\
\hline Moderate need & $90(22.5)$ & $60(66.7)$ & $30(33.3)$ & 0.74 & $42(21)$ & $48(24)$ & 0.47 \\
\hline Definite need & $99(24.7)$ & $59(59.6)$ & $40(40.4)$ & 0.79 & $38(19)$ & $61(30.5)$ & 0.08 \\
\hline Increased overjet (>3.5 mm) & $145(36.3)$ & $71(49)$ & $74(51)$ & 0.08 & $93(46.5)$ & $52(26)$ & $0.000^{*}$ \\
\hline Contact point displacements $(>1 \mathrm{~mm})$ & $216(54)$ & $131(60.6)$ & $85(39.4)$ & 0.345 & $87(43.5)$ & $129(65)$ & $0.000^{*}$ \\
\hline Increased overbite $(>3.5 \mathrm{~mm})$ & $105(26.3)$ & $55(52.4)$ & $50(47.6)$ & 0.138 & $62(31)$ & $43(21.5)$ & $0.03^{*}$ \\
\hline Crossbite & $91(22.8)$ & $57(62.6)$ & $34(37.4)$ & 0.362 & $30(15)$ & $61(30.5)$ & $0.000^{*}$ \\
\hline
\end{tabular}

${ }^{*}$ Significant differences in $t$-test results with $95 \%$ confidence interval.

TABLE 2: Prevalence of malocclusions in the total sample according to the level of need for orthodontic treatment.

\begin{tabular}{|c|c|c|c|c|c|}
\hline \multirow[b]{2}{*}{ Malocclusion } & \multicolumn{5}{|c|}{ Treatment need-DHC } \\
\hline & $N(\%)$ & $\begin{array}{c}\text { No/little } \\
N(\%)\end{array}$ & $\begin{array}{c}\text { Moderate } \\
N(\%)\end{array}$ & $\begin{array}{c}\text { Definite } \\
N(\%)\end{array}$ & $P$ value \\
\hline Increased overjet $(>3.5 \mathrm{~mm})$ & $145(36.3)$ & $69(47.5)$ & $33(22.8)$ & $43(29.7)$ & $0.036^{*}$ \\
\hline Contact point displacements (>1 mm) & $216(54.0)$ & $47(21.8)$ & $80(37.0)$ & $89(41.2)$ & $0.000^{*}$ \\
\hline Increased overbite $(>3.5)$ & $105(26.3)$ & $39(37.1)$ & $32(30.5)$ & $34(32.4)$ & $0.001^{*}$ \\
\hline Crossbite & $91(22.8)$ & $16(17.6)$ & $31(34.1)$ & $44(48.3)$ & $0.000^{*}$ \\
\hline
\end{tabular}

${ }^{*}$ Significant difference in chi-square test with $95 \%$ confidence interval.

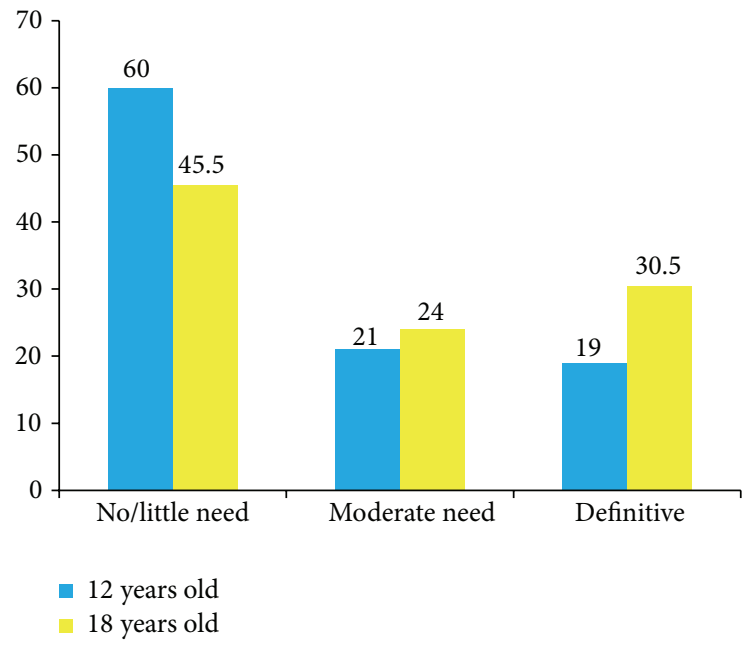

FIGURE 1: Frequency of grades DHC by age groups.

was identified between the age groups. Several studies in Asia have now reported on the prevalence of the need for treatment among the 12- to 18-year-old age group. For example, a previous study indicated that $70 \%$ of 12 -year-old Hong Kong Chinese school children had an objective need for treatment (grades 3-5) [5]; a study conducted in Shiraz, Iran, reported a prevalence of 51.7\% [7]; an examination of 12-yearolds in Malaysia recorded $47.9 \%$ [4], while the present study suggests that the need for orthodontic treatment is $47.2 \%$. This percentage score is higher than that recorded for the Nigerian population [8] and in Valencia, Spain [9].

With regard to the distribution of AC-IOTN, a definitive need (grades 8-10) was identified among 6.5\% of the subjects, a moderate need (grades 5-7) among $16.3 \%$, and $77.2 \%$ of the sample showed little need for treatment (grades 1-4). The need for orthodontic treatment among the Vietnamese sample did vary significantly by gender. Male subjects can have greater maxillary dimension widths than female subjects, and the crowding of anterior teeth is more likely among girls than boys. Similar results were obtained in a study conducted on the school population of Zahedan, Iran [10], and among Brazilian school children [11]. However, the category of definitive need according to AC recorded in the present study was higher than in the study of Ngom et al. [12] and lower than that recorded by Abdullah and Rock [4]. 
There was a low agreement between DHC and AC in this study because DHC in the present study was evaluated by occlusal anomalies while evaluating $\mathrm{AC}$, and the use of photographs of dentition limits overjet and lip-incisor evaluations [13]. Similar results had been found previously $[14,15]$. However, AC assesses the aesthetic aspects of malocclusion and plays a role in the sociopsychological impact of malocclusions for young people [16]. Class III malocclusion is a challenging orthodontic problem that is common in the Asian population $[17,18]$. According to Angle's classification in this study, the prevalence of class I, II, and III malocclusion was $67 \%, 17.5 \%$, and $15.5 \%$, respectively. We found an increasing prevalence of class III malocclusion between age groups from $10.5 \%$ among the 12 -year-olds to $20.5 \%$ for the 18 -year-olds. The results also showed that $66.1 \%$ of subjects had a treatment need according to DHC in the group of class III malocclusion $(P<0.001)$. The result revealed a statistically significant difference between age groups for class III malocclusion, increased overjet, contact point displacement, increased overbite, and crossbite $(P<$ 0.05). The prevalence of class III malocclusion, contact point displacement, and crossbite was greater among 18-year-olds than among 12-year-olds, while the prevalence of increased overjet and increased overbite was lower for 12-year-olds than among the 18-year-old group. This finding is in line with that of De Baets et al. [11]. Significant changes in total mandibular length continue into young adulthood with increases between late maturation stages $[15,19]$. In this study, for the 18-yearold group, increasing mandibular length leads to the lower first molars moving forward and consequently to an increase in class III malocclusion, compared to the prevalence among 12 -year-olds. In addition, all teeth were present in the dental arch after 12 years of age, which can cause a lack of space for teeth and increased crowding and also lead to teeth moving in the frontal direction, causing crossbite.

\section{Conclusions}

According to the DHC of the IOTN, $47.2 \%$ of the 12 -year-old school children and 18-year-old young adult population of Da Nang are in need of orthodontic treatment, as indicated by the proportions of contact point displacement, crossbite, increased overjet, and increased overbite among that group.

\section{Conflict of Interests}

The authors declare that they have no conflict of interests regarding the publication of this paper.

\section{Acknowledgment}

The authors thank the staff of the Faculty of Stomatology and the Human Research Ethics Committee of the Da Nang University of Medical Technology and Pharmacy, Vietnam, for supporting this project.

\section{References}

[1] N. C. Cons, J. Jenny, and F. J. Kohout, DAI: The Dental Aesthetic Index, College of Dentistry, University of Iowa, Iowa City, IA, USA, 1986.

[2] W. S. Parker, "The HLD (CalMod) index and the index question," American Journal of Orthodontics and Dentofacial Orthopedics, vol. 114, no. 2, pp. 134-141, 1998.

[3] P. H. Brook and W. C. Shaw, "The development of an index of orthodontic treatment priority," European Journal of Orthodontics, vol. 20, pp. 309-320, 1989.

[4] M. S. B. Abdullah and W. P. Rock, "Assessment of orthodontic treatment need in 5,112 Malaysian children using the IOTN and DAI indices," Community Dental Health, vol. 18, no. 4, pp. 242248, 2001.

[5] G. Wang, U. Hagg, and J. Ling, "The orthodontic treatment need and demand of Hong Kong Chinese children," The Chinese Journal of Dental Research, vol. 2, no. 3-4, pp. 84-92, 1999.

[6] C. Livas and K. Delli, "Subjective and objective perception of orthodontic treatment need: a systematic review," European Journal of Orthodontics, vol. 35, no. 3, pp. 347-353, 2013.

[7] S. M. Danaei, M. Oshagh, M. O. Khoda, A. A. Karamifar, S. S. Maram, and Z. Borhan-Haghighi, "Orthodontic treatment needs assessment of 14- to 18-year-old students in Shiraz, Iran.," World journal of orthodontics, vol. 11, no. 4, pp. 19-25, 2010.

[8] K. A. Kolawole, O. D. Otuyemi, S. O. Jeboda, and A. A. Umweni, "The need for orthodontic treatment in a school and referred population of Nigeria using the index of orthodontic treatment need (IOTN)," Odonto-Stomatologie Tropicale, vol. 31, no. 122, pp. 11-19, 2008.

[9] D. Manzanera, J. M. Montiel-Company, J. M. Almerich-Silla, and J. L. Gandía, "Orthodontic treatment need in Spanish schoolchildren: an epidemiological study using the Index of Orthodontic Treatment Need," European Journal of Orthodontics, vol. 31, no. 2, pp. 180-183, 2009.

[10] F. Shahri, S. R. Fakour, and S. Tabatabaee, "Use of the index of orthodontic treatment need in a school population of Zahedan," Life Science Journal, vol. 10, no. 2, pp. 240-244, 2013.

[11] P. F. Dias and R. Gleiser, "Orthodontic treatment need in a group of 9-12-year-old Brazilian schoolchildren," Brazilian Oral Research, vol. 23, no. 2, pp. 182-189, 2009.

[12] P. I. Ngom, F. Diagne, F. Dieye, K. Diop-Ba, and F. Thiam, "Orthodontic treatment need and demand in Senegalese school children aged 12-13 years: an appraisal using IOTN and ICON," Angle Orthodontist, vol. 77, no. 2, pp. 323-330, 2007.

[13] H. W. Fields, W. F. Vann Jr., and K. W. L. Vig, "Reliability of soft tissue profile analysis in children," Angle Orthodontist, vol. 52, no. 2, pp. 159-165, 1982.

[14] A. Borzabadi-Farahani, "Agreement between the index of complexity, outcome, and need and the dental and aesthetic components of the index of orthodontic treatment need," The American Journal of Orthodontics and Dentofacial Orthopedics, vol. 140, no. 2, pp. 233-238, 2011.

[15] F. Chen, K. Terada, and K. Hanada, "A special method of predicting mandibular growth potential for class III malocclusion," Angle Orthodontist, vol. 75, no. 2, pp. 191-195, 2005.

[16] E. De Baets, H. Lambrechts, J. Lemiere, L. Diya, and G. Willems, "Impact of self-esteem on the relationship between orthodontic treatment need and oral health-related quality of life in 11- to 16-year-old children," European Journal of Orthodontics, vol. 34, no. 6 , pp. 731-737, 2012. 
[17] K. K. Lew and W. C. Foong, "Horizontal skeletal typing in an ethnic Chinese population with true class III malocclusions," The British Journal of Orthodontics, vol. 20, no. 1, pp. 19-23, 1993.

[18] H. H. Lim, Y. J. Yoon, and K. W. Kim, "A study of characteristics of craniofacial skeleton on orthognathic surgical cases with skeletal Class III malocclusion," Korean Journal Orthodontics, vol. 28, pp. 189-201, 1998.

[19] T. Baccetti, B. C. Reyes, and J. A. McNamara Jr., "Craniofacial changes in Class III malocclusion as related to skeletal and dental maturation," The American Journal of Orthodontics and Dentofacial Orthopedics, vol. 132, no. 2, pp. 171.el-178.el, 2007. 


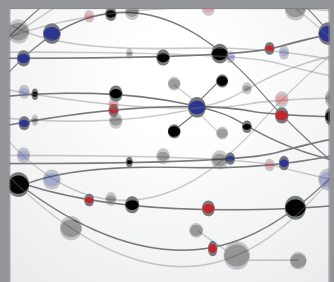

The Scientific World Journal
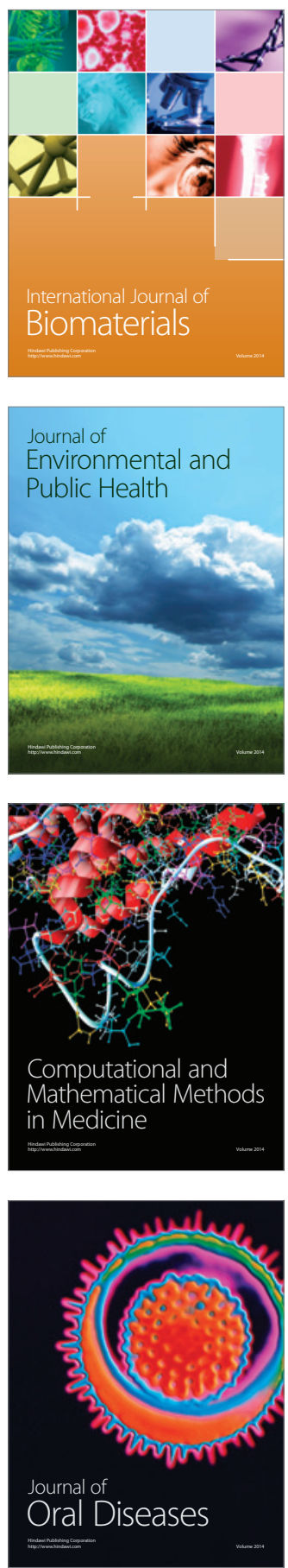
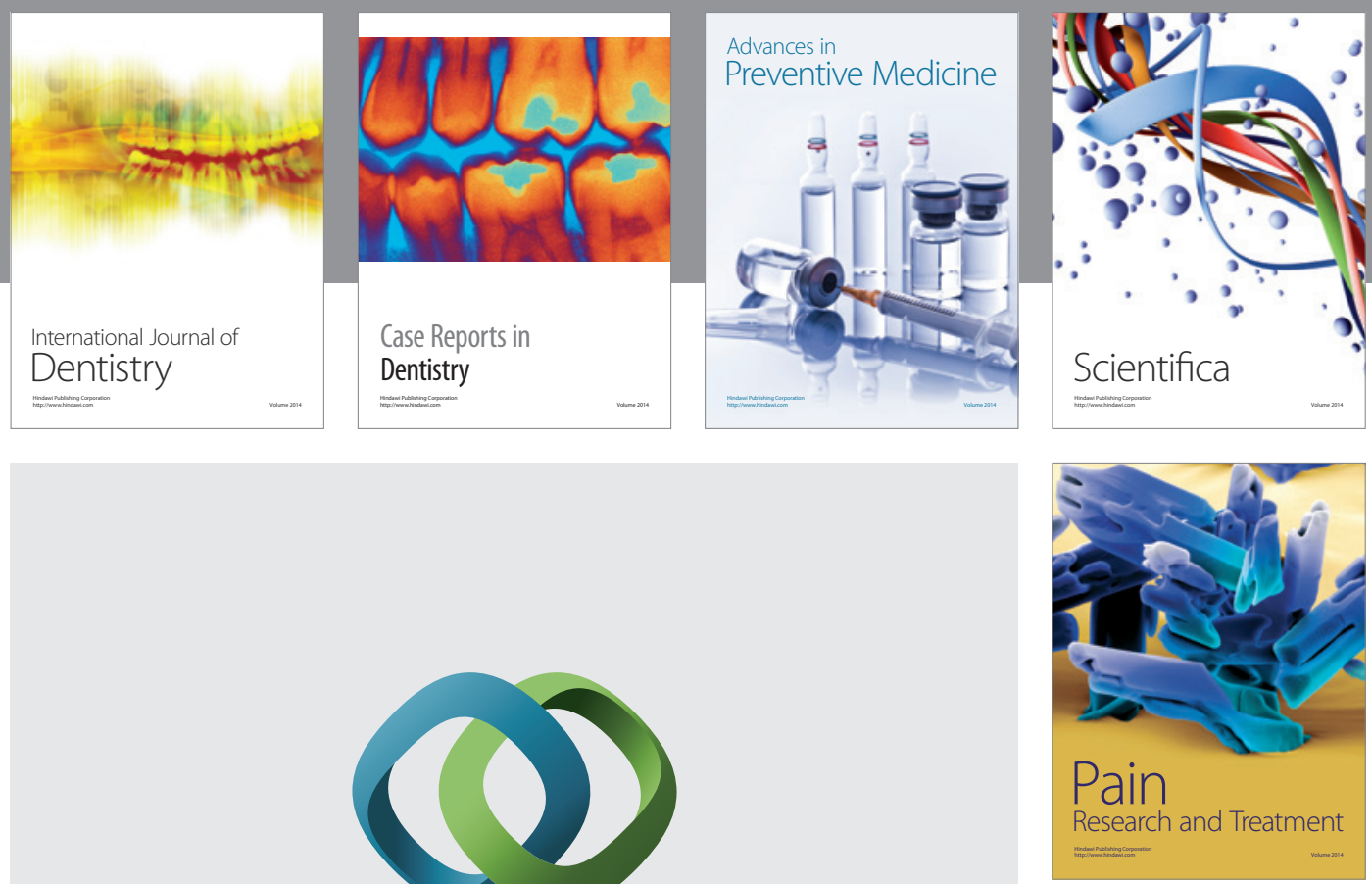

\section{Hindawi}

Submit your manuscripts at

http://www.hindawi.com
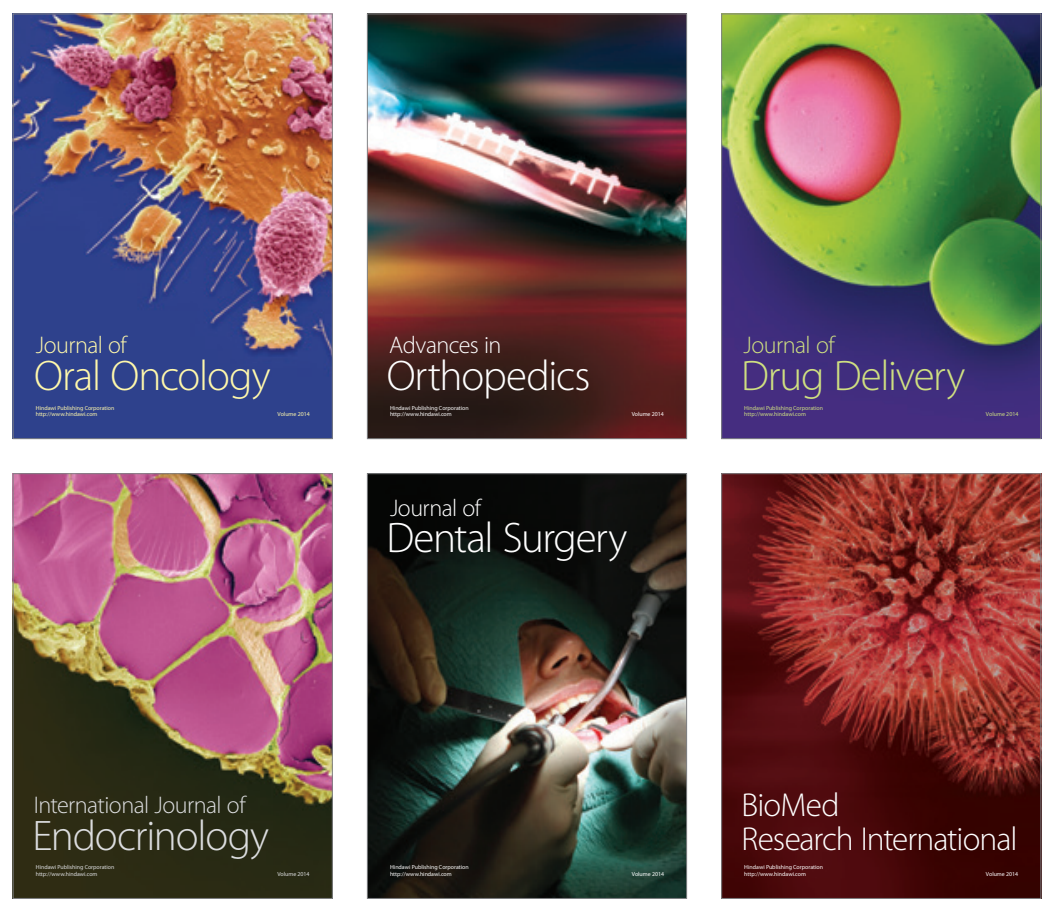

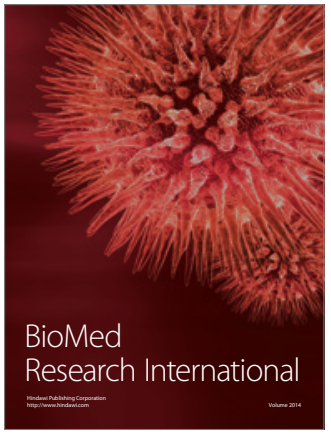

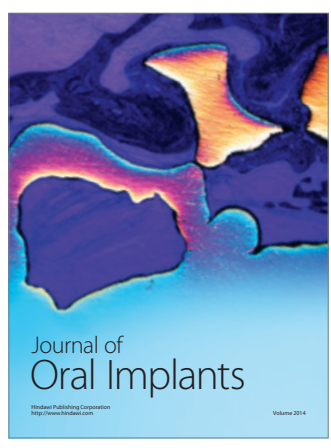
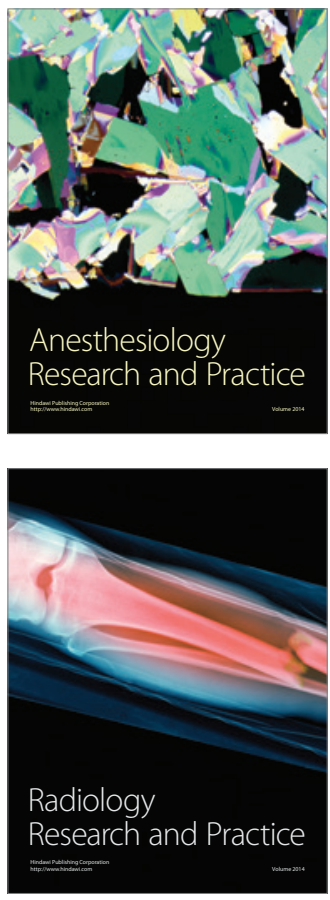